
\title{
$\begin{array}{ll}\text { Research Square } & \begin{array}{l}\text { Preprints are preliminary reports that have not undergone peer review. } \\ \text { They should not be considered conclusive, used to inform clinical practice, } \\ \text { or referenced by the media as validated information. }\end{array}\end{array}$
}

\section{The Volume Alterations of the Amygdala in Autism Spectrum Disorder: A Meta-Analysis on MRI Study}

Yunhua Chen

Department of Anatomy,School of Basic Medicine,Guizhou Medical University https://orcid.org/0000-0003-3659-3170

Xiaoli Zheng

State Key Laboratory of Functions and Applications of Medicinal Plants,Guizhou Medical University,Guizhou Provincial Engineering Technology Research Center for Chemical Drug R\&D

xue wang

Ultrasonic Center of Affiliated Hospital of Guizhou Medical University

Min Wen (D670418077@qq.com)

State Key Laboratory of Functions and Applications of Medicinal Plants,Guizhou Medical University,Guizhou Provincial Engineering Technology Research Center for Chemical Drug R\&D

Bo Zhou

State Key Laboratory of Functions and Applications of Medicinal Plants,GuiZhou Medical University, Guizhou Provincial Engineering Technology Research Center for Chemical Drug R\&D

\section{Research Article}

Keywords: Autism spectrum disorder, amygdala, MRI, meta-analysis

Posted Date: August 13th, 2021

DOI: https://doi.org/10.21203/rs.3.rs-757558/v1

License: (c) (i) This work is licensed under a Creative Commons Attribution 4.0 International License. Read Full License 


\section{Abstract}

Background:Due to the unclear etiology and pathogenesis of autism spectrum disorder (ASD), there is still a lack of effective drugs for prevention and treatment of ASD, which can improve the prognosis of autism through early intervention. However, the current diagnostic methods are mainly based on qualitative indicators such as behavioral standards, which cannot timely and accurately diagnose autism. A large number of studies have found abnormal amygdala volume in autism patients. This study aimed to evaluate amygdala volume in patients with ASD and elucidat the potential relationship between amygdala volume and ASD by using systematic review and meta-analysis, with a view to providing a new approach for early diagnosis of ASD.

Methods: Comprehensive electronic search in Pubmed, Web of Science, EBSCOhost, Embase, the Cochrane Review Library, CNKI and WanFang data to find original articles about the relationship between amygdala volume and the ASD. Heterogeneity across studies were assessed using $\mathrm{I}^{2}$ and Q statistics. Randomeffects model was used to estimate the odds ratios (ORs) with $95 \%$ confidence intervals (Cls). Sensitivity analyses to assess the influence of individual studies on the pooled estimate. Publication bias was investigated using funnel plots and Egger's regression test. If the funnel plot is asymmetry, the reason is distinguished by using the contour-enhanced funnel plots and trim-and-fill method.

Results: The results showed that the volume of the right amygdala significantly increased in patients with ASD (WMD: 0.137, 95\% Cl: [0.051, 0.222], Z = 3.132, $\mathrm{P}<0.001)$. Subgroup analysis by age showed that the volume of the left amygdala significantly increased in young child patients with ASD (MD: $0.153,95 \%$ Cl: $[0.025,0.281], Z=2.341, P<0.05)$, but not change in child (MD: 0.153, 95\% Cl: [-0.026, 0.332], Z = 1.672, P > 0.05), adolescent (MD: 0.095, 95\% Cl: [-0.189, $0.379], Z=0.654, P>0.05$ ) and adult (MD: $0.068,95 \% \mathrm{Cl}:[-0.103,0.240], Z=0.781, P>0.05)$ ASD patient. The volume of the right amygdala significantly increased in young child (MD: 0.174, 95\% Cl: [0.043, 0.304], Z = 2.60, $\mathrm{P}<0.05)$ and child patients(MD: 0.192, 95\% Cl: [0.040, 0.343], Z = 2.480, P< 0.05) with ASD, but not change in adolescent (MD: 0.208, 95\% Cl: [-0.201, 0.618], Z = 0.998, P > 0.05) and adulthood patients (MD: 0.126, 95\% Cl: [-0.040, 0.291], Z = $1.488, P>0.05)$. Subgroup analysis by gender showed that the volume of the left amygdala not change in male (MD: $0.101,95 \% \mathrm{Cl}:[-0.031,0.233], Z=1.498$, $P>0.05$ ) and female group (MD: $0.067,95 \% \mathrm{Cl}$ : $[-0.023,0.157], Z=1.452, P>0.05)$. The volume of the right amygdala significantly increased in female group (MD: $0.099,95 \% \mathrm{Cl}:[0.019,0.178], \mathrm{Z}=2.439, \mathrm{P}<0.05)$, but not change in male group (MD: $0.117,95 \% \mathrm{Cl}:[-0.014,0.248], \mathrm{Z}=1.755, \mathrm{P}>0.05)$.

Conclusions: The volume of the amygdala significantly increased in young children with ASD. These findings suggest that the volume of the amygdala in young children might be a potential early auxiliary imaging diagnosis method of ASD.

\section{Lay Summary}

The volume of the amygdala significantly increased in young children with ASD. These findings suggest that the volume of the amygdala in young children might be a potential early auxiliary imaging diagnosis method of ASD.

\section{Introduction}

Autism spectrum disorder (ASD) is a neurodevelopmental disorder characterized by impaired social interaction and communication as well as repetitive behaviors. ASD affects 14.7 per 1,000 children in the United States[1] and 4.1 per 1,000 in China[2]. Besides, ASD is more prevalence in males than in females, with reported ratios ranging from $2: 1$ to $5: 1[3,4]$. Early diagnosis of ASD is of paramount importance as it opens the road to early intervention, which is associated with better prognosis. However, most children with ASD are not diagnosed until preschool or school age, which means they are missing the most opportune time to be getting treatment. Therefore, it is very important to find a simple and non-invasive diagnostic method.

Although the precise neurobiological mechanism of behavioral social problems in autism is not yet fully understood, one influential hypothesis is that abnormal in the structures and/or functions of the "social brain area", particularly the prefrontal cortex, middle temporal gyrus and amygdala, underlie social malfunctioning in individuals with ASD[5-7]. As a hub in the "social brain area", the amygdala, which was located within the anterior medial portion of the brain's temporal lobe and included in the limbic system, maintains diverse connections with the neocortex, basal ganglia, hippocampus, thalamus and other brain cortical areas via a network of white matter (WM) tracts[8], responsible for information processing that subserve emotional learning, social cognition, and social interaction[9]. So, the emotional, behavioral, and social impairments in autism may be associated with the structural and/or functional abnormalities of the amygdala.MRI study of the amygdala in children with social interaction problems has found that social interaction problems are associated with the enlargement of the amygdala[10-12]. The size of the amygdala in autistic children increases during young childhood and does not change significantly in older children.In adults it shrinks. This is different from the amygdala development in healthy children[13, 14]. Many studies have shown that structural and/or functional abnormalities in the amygdala contribute to social dysfunction in ASD [8, 12, 13, 15-17]. The magnetic resonance imaging (MRI) technique, with its advantages of noninvasiveness, high spatial resolution, and improved contrast for soft tissues, has been widely applied for studying the brain diseases. Compare the volume change of the amygdala by MRI may provide a novel approach for the early diagnosis of autism, which is the key for early behavioral interference and individualized therapy. A considerable body of MRI studies have been conducted and found volume abnormal of amygdala in autism, but the results were not consistent. Some studies have found decreased volumes of amygdala in autism[18, 19], while others have found increased volumes in autism[2, 20]; and some studies didn't find differences[21, 22]. To evaluate more precisely the potential relationship between amygdala volume and the autism, further compare the differences of amygdala volume between different ages and genders, we hereby report on a meta-analysis using all available published data.

\section{Materials And Methods}

\section{Search Strategies}


A computerized search of Pubmed, Web of Science, EBSCOhost, Embase, the Cochrane Review Library, China National Knowledge Infrastructure (CNKI) and WanFangdata up to Nov 2020 was conducted using the following search strategy: ("autism" or "ASD" or "autism spectrum disorder"), and ("amygdala"), and ("MRI" or "Magnetic Resonance Imaging"). The search was restricted to English and Chinese language publications. A manual search of the references in the retrieved articles and relevant reviews was also conducted. A flowchart of information pertaining to identification, screening, eligibility, and the final selected datasets was constructed in accordance with the Preferred Reporting Items for Systematic Reviews and Meta-analyses (PRISMA) guidelines[23].

\section{Inclusion and Exclusion Criteria}

Studies comparing the volume of the amygdala between patients with autism and control subjects were included. The inclusion criteria were (1) Case-control study design; (2) demonstrated specific diagnostic criteria of autism; and (3) The means and standard deviations of amygdala volume (left or right) which derived from structural MRI was available in the report. The major criteria for exclusion were: (1) duplication of earlier publications (for studies using the same sample in different publications, only the most complete information was included following careful examination), (2) Animal studies, postmortem and voxelbased morphometry analyses were excluded. (3) The data of amygdala volume comes from a public database. (4) Unpublished papers, dissertations, editorials, conference articles, reviews and book chapters.

\section{Data Extraction}

The full texts of potentially relevant studies were then retrieved and assessed for eligibility by established criteria detailed above. The following data were also collected: author, year of publication, diagnostic criteria, country, sample size, mean age, gender, measuring method, and the mean, standard deviation of amygdala volume. All data were evaluated independently by two authors. Any disagreement about literature search, study selection, and data extraction was resolved by consensus under the guidance of the senior authors.

\section{Data Analysis}

All statistical analyses were performed using Weighted Mean Differences (WMD) methodology in STATA 15.0 (StataCorp, College Station, TX, United States). We used $95 \%$ confidence interval $(\mathrm{Cl})$ to gauge the precision of the summary estimates. Heterogeneity among the included studies was assessed using the $\mathrm{I}^{2}$ and Q statistic. A random-effects model or fixed-effects model was used to calculate the pooled WMD in the presence or absence of heterogeneity, respectively. The significance of the pooled MD was determined through a Z-test, and $p<0.05$ was considered to be statistically significant. Publication bias was investigated using funnel plots and Egger's regression test.The principle of Egger's regression test is the effect value that calculates each research, variance and standard error, with standard independent variable, effect value is linear regression.Its intercept represents the degree of asymmetry, and the greater the deviation from 0 , the more pronounced the degree of asymmetry.Egger's regression test and funnel plot were used to judge the publication bias from both quantitative and qualitative aspects.If the funnel plot is asymmetry, the reason for the asymmetry is distinguish by using the contour-enhanced funnel plots $[24,25]$ and trim-and-fill method[26, 27]. We also conducted sensitivity analysis to test the robustness of associations by sequentially omitting each of the included studies one at a time. To further explore the potential relationship between amygdala volume and ages and genders of autism, subgroup analyses were performed.

\section{Results}

\subsection{Literature Search and Study Characteristics}

We used several search criteria to include or exclude reported studies on the relationship between the volume of the amygdala and autism (Fig. 1). A total of 28 studies $[2,10,13,18-22,28-47]$ (1,011 cases and 823 controls) concerning the volume of the amygdala were included in the final evaluation (Table 1$)$. 
Table 1

Main characteristics of datasets included in this meta-analysis

\begin{tabular}{|c|c|c|c|c|c|c|c|c|c|c|c|}
\hline \multirow[t]{2}{*}{ No. } & \multirow[t]{2}{*}{ First Author } & \multirow[t]{2}{*}{ Year } & \multirow[t]{2}{*}{ Country } & \multirow[t]{2}{*}{$\begin{array}{l}\text { Diagnostic } \\
\text { criteria }\end{array}$} & \multirow[t]{2}{*}{ Sample size } & \multirow[t]{2}{*}{ Gender } & \multirow{2}{*}{$\begin{array}{l}\text { Age } \\
\text { (year) }\end{array}$} & \multirow{2}{*}{$\begin{array}{l}\text { MRI techniques/ } \\
\text { Segmentation }\end{array}$} & \multirow{2}{*}{$\begin{array}{l}\text { Total } \\
\text { brain } \\
(\mathrm{cm} 3)\end{array}$} & \multicolumn{2}{|c|}{$\begin{array}{l}\text { Amygdala } \\
\text { volume (cm3) }\end{array}$} \\
\hline & & & & & & & & & & Left & right \\
\hline \multirow[t]{2}{*}{1} & \multirow[t]{2}{*}{ Haznedar } & \multirow[t]{2}{*}{2000} & \multirow[t]{2}{*}{ American } & \multirow[t]{2}{*}{ DSM-IV } & $\begin{array}{l}\text { ASD }(n= \\
17, M / F: 15 / 2)\end{array}$ & \multirow[t]{2}{*}{ MIX } & $\begin{array}{l}27.7 \pm \\
11.3\end{array}$ & \multirow[t]{2}{*}{$\begin{array}{l}\text { 1.5 T GE } \\
\text { Signa/Manual }\end{array}$} & $\begin{array}{l}1314 \pm \\
122\end{array}$ & $\begin{array}{l}1.21 \\
+ \\
0.22\end{array}$ & $\begin{array}{l}1.217+ \\
0.267\end{array}$ \\
\hline & & & & & $\begin{array}{l}\operatorname{TDC}(\mathrm{n}= \\
31, \mathrm{M} / \mathrm{F}: 15 / 2)\end{array}$ & & $\begin{array}{l}28.8 \pm \\
9.4\end{array}$ & & $\begin{array}{l}1304 \pm \\
178\end{array}$ & $\begin{array}{l}1.22 \\
+ \\
0.22\end{array}$ & $\begin{array}{l}1.215+ \\
0.258\end{array}$ \\
\hline \multirow[t]{2}{*}{2} & \multirow[t]{2}{*}{ Pierce } & \multirow[t]{2}{*}{2001} & \multirow[t]{2}{*}{ American } & \multirow[t]{2}{*}{ DSM-IV } & ASD $(n=7)$ & \multirow[t]{2}{*}{ M } & $21-41$ & \multirow[t]{2}{*}{$\begin{array}{l}\text { 1.5 T GE } \\
\text { Signa/Manual }\end{array}$} & $\begin{array}{l}\text { Not } \\
\text { report }\end{array}$ & $\begin{array}{l}0.92 \\
\pm \\
0.13\end{array}$ & $\begin{array}{l}0.87 \pm \\
0.12\end{array}$ \\
\hline & & & & & $\operatorname{TDC}(\mathrm{n}=8)$ & & $20-42$ & & $\begin{array}{l}\text { Not } \\
\text { reportNot } \\
\text { report }\end{array}$ & $\begin{array}{l}1.09 \\
\pm \\
0.13\end{array}$ & $\begin{array}{l}1.01 \pm \\
0.11\end{array}$ \\
\hline \multirow[t]{6}{*}{3} & \multirow[t]{6}{*}{ Sparks } & \multirow[t]{6}{*}{2002} & \multirow[t]{6}{*}{ American } & \multirow[t]{6}{*}{$\begin{array}{l}\text { DSM-IV, } \\
\text { ADOS-G }\end{array}$} & $\begin{array}{l}\text { All ASD }(n= \\
45)\end{array}$ & MIX & $\begin{array}{l}3.17- \\
4.50\end{array}$ & \multirow[t]{6}{*}{$\begin{array}{l}\text { 1.5 T GE } \\
\text { Signa/Automated }\end{array}$} & $\begin{array}{l}1191.87 \\
\pm 94.71\end{array}$ & $\begin{array}{l}1.25 \\
\pm \\
0.23\end{array}$ & $\begin{array}{l}1.19 \pm \\
0.26\end{array}$ \\
\hline & & & & & $\begin{array}{l}\text { All TDC }(n= \\
26)\end{array}$ & MIX & $\begin{array}{l}3.0- \\
4.67\end{array}$ & & $\begin{array}{l}1085.87 \\
\pm 109.2\end{array}$ & $\begin{array}{l}1.10 \\
\pm \\
0.20\end{array}$ & $\begin{array}{l}1.02 \pm \\
0.23\end{array}$ \\
\hline & & & & & $\operatorname{ASD}(n=38)$ & M & & & & $\begin{array}{l}1.25 \\
\pm \\
0.23\end{array}$ & $\begin{array}{l}1.20 \pm \\
0.25\end{array}$ \\
\hline & & & & & $\operatorname{TDC}(\mathrm{n}=18)$ & & & & & $\begin{array}{l}1.08 \\
\pm \\
0.20\end{array}$ & $\begin{array}{l}0.94 \pm \\
0.19\end{array}$ \\
\hline & & & & & ASD $(n=7)$ & $\mathrm{F}$ & & & & $\begin{array}{l}1.20 \\
\pm \\
0.22\end{array}$ & $\begin{array}{l}1.14 \pm \\
0.29\end{array}$ \\
\hline & & & & & $\operatorname{TDC}(\mathrm{n}=8)$ & & & & & $\begin{array}{l}1.16 \\
\pm \\
0.21\end{array}$ & $\begin{array}{l}1.18 \pm \\
0.22\end{array}$ \\
\hline \multirow[t]{2}{*}{4} & Rojas & 2004 & American & DSM-IV & ASD $(n=15)$ & MIX & $19-47$ & $\begin{array}{l}\text { 1.5 T GE } \\
\text { Signa/Manual }\end{array}$ & $\begin{array}{l}1237.57 \\
\pm 166.27\end{array}$ & $\begin{array}{l}2.46 \\
\pm \\
0.41\end{array}$ & $\begin{array}{l}2.57 \pm \\
0.43\end{array}$ \\
\hline & & & & & $\operatorname{TDC}(n=17)$ & & $37-51$ & & $\begin{array}{l}1257.87 \\
\pm 87.69\end{array}$ & $\begin{array}{l}2.57 \\
\pm \\
0.24\end{array}$ & $\begin{array}{l}2.60 \pm \\
0.30\end{array}$ \\
\hline 5 & *Schumann & 2004 & American & $\begin{array}{l}\text { ADI-R and } \\
\text { ADOS-G }\end{array}$ & $\begin{array}{l}\text { All ASD }(n= \\
63)\end{array}$ & M & $\begin{array}{l}12.7 \pm \\
3.5\end{array}$ & $\begin{array}{l}1.5 \mathrm{~T} \text { and } 3.0 \mathrm{~T} \mathrm{GE} \\
\text { Signa/Manual }\end{array}$ & $\begin{array}{l}1214 \pm \\
119.3\end{array}$ & $\begin{array}{l}2.05 \\
\pm \\
0.22\end{array}$ & $\begin{array}{l}2.13 \pm \\
0.23\end{array}$ \\
\hline & & & & & $\begin{array}{l}\text { All TDC }(\mathrm{n}= \\
22)\end{array}$ & M & $\begin{array}{l}13.1 \pm \\
3.1\end{array}$ & & $\begin{array}{l}1190 \pm \\
77\end{array}$ & $\begin{array}{l}1.94 \\
\pm \\
0.30\end{array}$ & $\begin{array}{l}1.96 \pm \\
0.30\end{array}$ \\
\hline & & & & & ASD $(n=30)$ & M & $\begin{array}{l}7.5- \\
12.5\end{array}$ & & & $\begin{array}{l}2.04 \\
\pm \\
0.25\end{array}$ & $\begin{array}{l}2.10 \pm \\
0.21\end{array}$ \\
\hline & & & & & $\operatorname{TDC}(\mathrm{n}=11)$ & M & $\begin{array}{l}7.5- \\
12.5\end{array}$ & & & $\begin{array}{l}1.77 \\
\pm \\
0.18\end{array}$ & $\begin{array}{l}1.81 \pm \\
0.18\end{array}$ \\
\hline & & & & & ASD $(n=33)$ & M & $\begin{array}{l}12.75- \\
18.5\end{array}$ & & & $\begin{array}{l}2.05 \\
\pm \\
0.23\end{array}$ & $\begin{array}{l}2.16 \pm \\
0.25\end{array}$ \\
\hline & & & & & $\operatorname{TDC}(\mathrm{n}=11)$ & M & $\begin{array}{l}12.75- \\
18.5\end{array}$ & & & $\begin{array}{l}2.11 \\
\pm \\
0.25\end{array}$ & $\begin{array}{l}2.10 \pm \\
0.22\end{array}$ \\
\hline 6 & Boucher & 2005 & UK & $\begin{array}{l}\text { WADIC } \\
\text { and DSM- } \square\end{array}$ & ASD $(n=10)$ & M & $23 \pm 9$ & $\begin{array}{l}1.5 \mathrm{~T} \mathrm{GE} \\
\text { Signa/Automated }\end{array}$ & $\begin{array}{l}1215.75 \\
\pm 37.55\end{array}$ & $\begin{array}{l}2.82 \\
\pm \\
0.14\end{array}$ & $\begin{array}{l}2.98 \pm \\
0.21\end{array}$ \\
\hline & & & & & $\operatorname{TDC}(\mathrm{n}=10)$ & & $24 \pm 2$ & & $\begin{array}{l}1127.25 \\
\pm 18.41\end{array}$ & $\begin{array}{l}2.34 \\
\pm 0.1\end{array}$ & $\begin{array}{l}2.41 \pm \\
0.12\end{array}$ \\
\hline 7 & Nacewicz & 2006 & American & ADI-R & $\operatorname{ASD}(n=16)$ & M & $\begin{array}{l}14.3 \pm \\
4.7\end{array}$ & $\begin{array}{l}\text { 3.0-T GE } \\
\text { Signa/Automated }\end{array}$ & $\begin{array}{l}\text { Not } \\
\text { report }\end{array}$ & $\begin{array}{l}1.78 \\
\pm\end{array}$ & $\begin{array}{l}1.770 \pm \\
0.123\end{array}$ \\
\hline
\end{tabular}




\begin{tabular}{|c|c|c|c|c|c|c|c|c|c|c|c|}
\hline & & & & & $\operatorname{TDC}(\mathrm{n}=14)$ & & $\begin{array}{l}13.7 \pm \\
3.9\end{array}$ & & $\begin{array}{l}\text { Not } \\
\text { report }\end{array}$ & $\begin{array}{l}1.92 \\
\pm \\
0.17\end{array}$ & $\begin{array}{l}1.921 \pm \\
0.186\end{array}$ \\
\hline \multirow[t]{2}{*}{8} & Palmen & 2006 & Netherlands & DSM-IV & ASD $(n=42)$ & MIX & $\begin{array}{l}15.6 \pm \\
5.25\end{array}$ & $\begin{array}{l}\text { 1.5 T GE } \\
\text { Signa/Manual }\end{array}$ & $\begin{array}{l}1408.36 \\
\pm 99.32\end{array}$ & $\begin{array}{l}1.32 \\
\pm \\
0.38\end{array}$ & $\begin{array}{l}1.51 \pm \\
0.27\end{array}$ \\
\hline & & & & & $\operatorname{TDC}(\mathrm{n}=42)$ & & $\begin{array}{l}15.33 \\
\pm 5.40\end{array}$ & & $\begin{array}{l}1345.59 \\
\pm 78.77\end{array}$ & $\begin{array}{l}1.29 \\
\pm \\
0.30\end{array}$ & $\begin{array}{l}1.53 \pm \\
0.29\end{array}$ \\
\hline \multirow[t]{2}{*}{9} & Corbett & 2009 & American & $\begin{array}{l}\text { DSM-IV, } \\
\text { ADOS }\end{array}$ & $\operatorname{ASD}(n=12)$ & MIX & $\begin{array}{l}9.63 \pm \\
1.48\end{array}$ & $\begin{array}{l}\text { 1.5 T GE } \\
\text { Signa/Manual }\end{array}$ & $\begin{array}{l}1299.78 \\
\pm 47.15\end{array}$ & $\begin{array}{l}1.76 \\
\pm \\
0.31\end{array}$ & $\begin{array}{l}1797.38 \\
\pm 356.30\end{array}$ \\
\hline & & & & & $\operatorname{TDC}(\mathrm{n}=15)$ & & $\begin{array}{l}9.63 \pm \\
1.47\end{array}$ & & $\begin{array}{l}1247.05 \\
\pm 79.91\end{array}$ & $\begin{array}{l}1.81 \\
\pm \\
0.22\end{array}$ & $\begin{array}{l}1811.62 \\
\pm 201.91\end{array}$ \\
\hline \multirow[t]{2}{*}{10} & Hazlett & 2009 & American & $\begin{array}{l}\text { ADI-R and } \\
\text { ADOS-G }\end{array}$ & $\operatorname{ASD}(n=63)$ & M & $\begin{array}{l}2.8 \pm \\
0.4\end{array}$ & $\begin{array}{l}\text { 1.5 T GE } \\
\text { Signa/Manual }\end{array}$ & $\begin{array}{l}\text { Not } \\
\text { report }\end{array}$ & $\begin{array}{l}1.92 \\
\pm \\
0.03\end{array}$ & $\begin{array}{l}2.03 \pm \\
0.03\end{array}$ \\
\hline & & & & & $\operatorname{TDC}(\mathrm{n}=31)$ & & $\begin{array}{l}2.6 \pm \\
0.6\end{array}$ & & $\begin{array}{l}\text { Not } \\
\text { report }\end{array}$ & $\begin{array}{l}1.58 \\
\pm \\
0.05\end{array}$ & $\begin{array}{l}1.67 \pm \\
0.05\end{array}$ \\
\hline \multirow[t]{6}{*}{11} & *Schumann & 2009 & American & $\begin{array}{l}\text { ADI-R and } \\
\text { DSM-IV }\end{array}$ & $\begin{array}{l}\text { All ASD }(n= \\
50)\end{array}$ & MIX & & $\begin{array}{l}\text { 1.5 T GE } \\
\text { Signa/Manual }\end{array}$ & $\begin{array}{l}\text { Not } \\
\text { report }\end{array}$ & $\begin{array}{l}1.66 \\
\pm \\
0.24\end{array}$ & $\begin{array}{l}1.72 \pm \\
0.23\end{array}$ \\
\hline & & & & & $\begin{array}{l}\text { All TDC }(\mathrm{n}= \\
39)\end{array}$ & MIX & & & $\begin{array}{l}\text { Not } \\
\text { report }\end{array}$ & $\begin{array}{l}1.57 \\
\pm \\
0.24\end{array}$ & $\begin{array}{l}1.61 \pm \\
0.24\end{array}$ \\
\hline & & & & & ASD $(n=38)$ & M & $\begin{array}{l}3.0 \pm \\
0.76\end{array}$ & & & $\begin{array}{l}1.75 \\
\pm \\
0.20\end{array}$ & $\begin{array}{l}1.81 \pm \\
0.19\end{array}$ \\
\hline & & & & & $\operatorname{TDC}(\mathrm{n}=28)$ & M & $\begin{array}{l}2.83 \pm \\
0.59\end{array}$ & & & $\begin{array}{l}1.69 \\
\pm \\
0.15\end{array}$ & $\begin{array}{l}1.73 \pm \\
0.16\end{array}$ \\
\hline & & & & & $\operatorname{ASD}(n=12)$ & $\mathrm{F}$ & $\begin{array}{l}4.67 \pm \\
0.51\end{array}$ & & & $\begin{array}{l}1.40 \\
\pm \\
0.14\end{array}$ & $\begin{array}{l}1.47 \pm \\
0.10\end{array}$ \\
\hline & & & & & $\operatorname{TDC}(\mathrm{n}=11)$ & $\mathrm{F}$ & $\begin{array}{l}3.08 \pm \\
0.53\end{array}$ & & & $\begin{array}{l}1.26 \\
\pm \\
0.07\end{array}$ & $\begin{array}{l}1.31 \pm \\
0.07\end{array}$ \\
\hline \multirow[t]{2}{*}{12} & Dziobek & 2010 & Germany & $\begin{array}{l}\text { ADI-R and } \\
\text { DSM-IV }\end{array}$ & $\operatorname{ASD}(n=27)$ & MIX & $\begin{array}{l}42.0 \pm \\
11.3\end{array}$ & $\begin{array}{l}\text { not } \\
\text { report/Automated }\end{array}$ & $\begin{array}{l}\text { Not } \\
\text { report }\end{array}$ & $\begin{array}{l}1.00 \\
\pm \\
0.15\end{array}$ & $\begin{array}{l}1.04 \pm \\
0.19\end{array}$ \\
\hline & & & & & $\operatorname{TDC}(\mathrm{n}=29)$ & & $\begin{array}{l}44.9 \pm \\
14.6\end{array}$ & & $\begin{array}{l}\text { Not } \\
\text { report }\end{array}$ & $\begin{array}{l}1.04 \\
\pm \\
0.15\end{array}$ & $\begin{array}{l}1.04 \pm \\
0.22\end{array}$ \\
\hline \multirow[t]{2}{*}{13} & Groen & 2010 & Netherlands & DSM-IV & ASD $(n=23)$ & MIX & $\begin{array}{l}15.15 \\
\pm 1.86\end{array}$ & $\begin{array}{l}\text { 1.5-T } \\
\text { Siemens/Automated }\end{array}$ & $\begin{array}{l}1683.26 \\
\pm 210.87\end{array}$ & $\begin{array}{l}1.69 \\
\pm \\
0.20\end{array}$ & $\begin{array}{l}1.756 \pm \\
0.200\end{array}$ \\
\hline & & & & & $\operatorname{TDC}(\mathrm{n}=29)$ & & $\begin{array}{l}15.65 \\
\pm 1.66\end{array}$ & & $\begin{array}{l}1637.9 \pm \\
185.14\end{array}$ & $\begin{array}{l}1.59 \\
\pm \\
0.16\end{array}$ & $\begin{array}{l}1.629 \pm \\
0.144\end{array}$ \\
\hline \multirow[t]{2}{*}{14} & kim & 2010 & Korea & DSM-IV & $\begin{array}{l}\text { ASD }(n= \\
31, M / F: 24 / 7)\end{array}$ & MIX & $6-7$ & $\begin{array}{l}\text { 1.5 T GE } \\
\text { Signa/Manual }\end{array}$ & $\begin{array}{l}\text { Not } \\
\text { report }\end{array}$ & $\begin{array}{l}1.82 \\
\pm \\
0.20\end{array}$ & $\begin{array}{l}1.68 \pm \\
0.25\end{array}$ \\
\hline & & & & & $\begin{array}{l}\text { TDC }(n= \\
20, M / F: 12 / 8)\end{array}$ & & $6-7$ & & $\begin{array}{l}\text { Not } \\
\text { report }\end{array}$ & $\begin{array}{l}1.60 \\
\pm \\
0.30\end{array}$ & $\begin{array}{l}1.45 \pm \\
0.27\end{array}$ \\
\hline \multirow[t]{4}{*}{15} & O'Brien & 2010 & UK & DSM-IV & $\begin{array}{l}\text { All ASD ( } \mathrm{n}= \\
22)\end{array}$ & MIX & & $\begin{array}{l}\text { 1.5 T GE } \\
\text { Signa/Manual }\end{array}$ & $\begin{array}{l}\text { Not } \\
\text { report }\end{array}$ & $\begin{array}{l}2.74 \\
\pm \\
0.47\end{array}$ & $\begin{array}{l}2.64 \pm \\
0.44\end{array}$ \\
\hline & & & & & $\begin{array}{l}\text { All TDC }(n= \\
22)\end{array}$ & & & & $\begin{array}{l}\text { Not } \\
\text { report }\end{array}$ & $\begin{array}{l}2.7 \\
\pm 0.3\end{array}$ & $\begin{array}{l}2.65 \pm \\
0.30\end{array}$ \\
\hline & & & & & $\operatorname{ASD}(n=12)$ & & $13 \pm 2$ & & & $\begin{array}{l}2.6 \\
\pm 0.5\end{array}$ & $2.5 \pm 0.5$ \\
\hline & & & & & $\operatorname{TDC}(\mathrm{n}=10)$ & & $13 \pm 2$ & & & 2.7 & $2.7 \pm 0.3$ \\
\hline
\end{tabular}




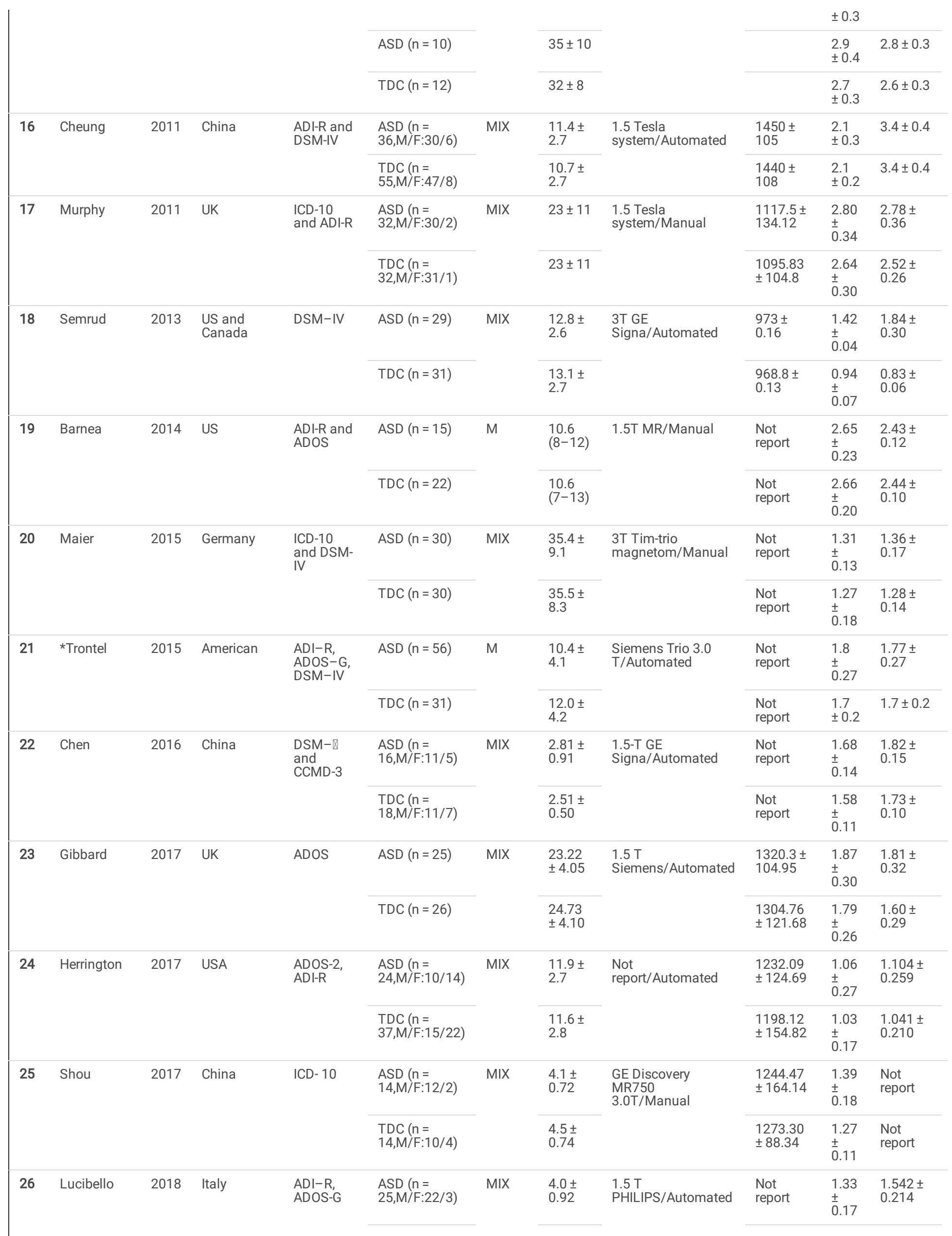




\begin{tabular}{|c|c|c|c|c|c|c|c|c|c|c|c|}
\hline & & & & & $\begin{array}{l}\text { TDC }(n= \\
28, M / F: 21 / 7)\end{array}$ & & $\begin{array}{l}4.42 \pm \\
0.75\end{array}$ & & $\begin{array}{l}\text { Not } \\
\text { report }\end{array}$ & $\begin{array}{l}1.31 \\
\pm \\
0.19\end{array}$ & $\begin{array}{l}1.490 \pm \\
0.227\end{array}$ \\
\hline \multirow[t]{2}{*}{27} & Zhu & 2018 & China & DSM IV & $\begin{array}{l}\text { ASD }(n= \\
39, M / F: 35 / 4)\end{array}$ & MIX & $\begin{array}{l}2.15 \pm \\
0.70\end{array}$ & 3.0T MR/Manual & $\begin{array}{l}\text { Not } \\
\text { report }\end{array}$ & $\begin{array}{l}1.04 \\
\pm \\
0.41\end{array}$ & $\begin{array}{l}1.09 \pm \\
0.38\end{array}$ \\
\hline & & & & & $\begin{array}{l}\text { TDC }(n= \\
24, M / F: 18 / 6)\end{array}$ & & $\begin{array}{l}3.64 \pm \\
1.08\end{array}$ & & $\begin{array}{l}\text { Not } \\
\text { report }\end{array}$ & $\begin{array}{l}0.67 \\
\pm \\
0.25\end{array}$ & $\begin{array}{l}0.75 \pm \\
0.19\end{array}$ \\
\hline \multirow[t]{6}{*}{28} & *Nordahl & 2020 & American & $\begin{array}{l}\text { ADI-R, } \\
\text { ADOS-G }\end{array}$ & $\begin{array}{l}\text { All ASD }(n= \\
226)\end{array}$ & MIX & & $\begin{array}{l}\text { 3T Siemens } \\
\text { Trio/Manual }\end{array}$ & $\begin{array}{l}\text { Not } \\
\text { report }\end{array}$ & $\begin{array}{l}1.56 \\
\pm \\
0.18\end{array}$ & $\begin{array}{l}1.73 \pm \\
0.19\end{array}$ \\
\hline & & & & & $\begin{array}{l}\text { All TDC }(\mathrm{n}= \\
120)\end{array}$ & MIX & & & $\begin{array}{l}\text { Not } \\
\text { report }\end{array}$ & $\begin{array}{l}1.51 \\
\pm \\
0.15\end{array}$ & $\begin{array}{l}1,64 \pm \\
0.16\end{array}$ \\
\hline & & & & & $\operatorname{ASD}(n=73)$ & $\mathrm{F}$ & $\begin{array}{l}3.25 \pm \\
0.53\end{array}$ & & & $\begin{array}{l}1.47 \\
\pm \\
0.17\end{array}$ & $\begin{array}{l}1.64 \pm \\
0.19\end{array}$ \\
\hline & & & & & $\operatorname{TDC}(\mathrm{n}=57)$ & $\mathrm{F}$ & $\begin{array}{l}3.16 \pm \\
0.5\end{array}$ & & & $\begin{array}{l}1.45 \\
\pm \\
0.13\end{array}$ & $\begin{array}{l}1.57 \pm \\
0.16\end{array}$ \\
\hline & & & & & $\operatorname{ASD}(n=153)$ & M & $\begin{array}{l}3.24 \pm \\
0.57\end{array}$ & & & $\begin{array}{l}1.61 \\
\pm \\
0.16\end{array}$ & $\begin{array}{l}1.78 \pm \\
0.17\end{array}$ \\
\hline & & & & & $\operatorname{TDC}(\mathrm{n}=63)$ & $M$ & $\begin{array}{l}3.07 \pm \\
0.49\end{array}$ & & & $\begin{array}{l}1.56 \\
\pm \\
0.15\end{array}$ & $\begin{array}{l}1.70 \pm \\
0.14\end{array}$ \\
\hline
\end{tabular}

\subsection{The volume of amygdala in autism versus control group}

\subsubsection{The volume of left amygdala}

28 studies (included 1011 ASD and 823 controls) were used in this analysis. These studies had significant heterogeneity $\left(\mathrm{I}^{2}=96.8 \%, 95 \% \mathrm{Cl}\right.$ : [82.3\%, 98.7\%]; $\mathrm{H}$ $=5.582,95 \% \mathrm{Cl}:[2.374,8.836]$, tau $\left.^{2}=0.0406\right)$, so a random-effect model was used to calculate pooled WMD. Forest plot (Fig. $\left.2 \mathrm{~A}\right)$ showed that the diamond was on the right side of the vertical line and did not intersect with the line, which demonstrates a significantly increase the volume of the left amygdala in patients with ASD compared to control (WMD: 0.095, 95\% Cl: [0.017, 0.174], Z = 2.379, $\mathrm{P}<0.05$ ). The funnel plot (Fig. 2B) was asymmetrical, as assessed by visual inspection and Egger's meta-regression analysis ( $p<0.001,95 \% \mathrm{Cl}$ : $[-7.79,-2.63]$, Fig. $2 \mathrm{C}$ ). To differentiating asymmetry due to publication bias from that due to other factors, the contour-enhanced funnel plot (Fig. 2D) was used. The missing data were found distributed in left side (shaded area, statistical significance), and no additional studies were found by the application of the trim-and-fill method, hence asymmetry might have been caused by several factors (such as true heterogeneity) and not by publication bias. In addition, we found that the asymmetry of funnel plot was caused by 4 articles[28, 37,39 , 43]. When these articles were removed, the heterogeneity was significantly reduced $\left(I^{2}=54.1 \%\right)$ and the diamond was extremely close to the vertical line (WMD: $0.042,95 \% \mathrm{Cl}$ : $[0.008,0.075)$. Sensitivity analysis suggested that the results were affected by Hazlett's study, when removed the article, the diamond was intersect with the vertical line.

\subsubsection{The volume of right amygdala}

27 studies (included 996 ASD and 809 controls) were used in this analysis. These studies had significant heterogeneity $\left(\mathrm{I}^{2}=96.2 \%, 95 \% \mathrm{Cl}\right.$ : [79.7\%, $\left.98.5 \%\right] ; \mathrm{H}=$ $\left.5.127,95 \% \mathrm{Cl}:[2.218,8.075], \mathrm{tau}^{2}=0.0469\right)$, so a random-effect model was used to calculate pooled WMD. Forest plot (Fig. $\left.3 \mathrm{~A}\right)$ showed that the diamond was on the right side of the vertical line and did not intersect with the line, which demonstrates a significantly increase the volume of the right amygdala in patients with ASD compared to control (WMD: 0.137, 95\% Cl: [0.051, 0.222], Z = 3.132, P<0.001). The funnel plot (Fig. 3B) was asymmetrical, as assessed by visual inspection of the plot and by Egger's meta-regression analysis ( $p=0.016<0.05,95 \% \mathrm{Cl}$ : $[-6.30,-0.75]$, Fig. $3 \mathrm{C}$ ). To differentiating asymmetry due to publication bias from that due to other factors, the contour-enhanced funnel plot (Fig. 3D) was used. The missing data were found distributed in left side (shaded area, statistical significance), and no additional studies were found by the application of the trim-and-fill method, hence asymmetry might have been caused by several factors (such as true heterogeneity) and not by publication bias. We also found that the asymmetry of funnel plot was caused by 4 articles[28, 37,39 , 43]. When these articles were removed, the heterogeneity was significantly reduced $\left(I^{2}=63.0 \%\right)$ and the diamond was still on the right side of the vertical line and did not intersect with the line (WMD: 0.061, 95\% Cl: [0.022, 0.099). Sensitivity analysis suggested that the results were robust and reliable.

\section{3 subgroup analysis}

\subsection{1 subgroup analysis by age}

To explore the relationship between age and amygdala volume, a subgroup analysis by age was conducted. We divided all studies into five groups according to age: (1) young children (0-6 years), (2) children (6-12 years), (3) adolescent (12-18 years), (4) adult (> 18 years) and (5) other group. Our results show that the volume of the left amygdala (Fig. 4A) significantly increase in young child patients with ASD (MD: $0.153,95 \%$ Cl: [0.025, 0.281], Z = 2.341, P<0.05), but not change in child (MD: $0.153,95 \% \mathrm{Cl}:[-0.026,0.332], Z=1.672, \mathrm{P}>0.05)$, adolescent (MD: $0.095,95 \% \mathrm{Cl}:[-0.189,0.379], Z=0.654, P>0.05)$ and adult (MD:

Page $7 / 16$ 
$0.068,95 \% \mathrm{Cl}:[-0.103,0.240], \mathrm{Z}=0.781, \mathrm{P}>0.05)$ ASD patient. The volume of the right amygdala (Fig. 4B) significantly increased in young child (MD: 0.174 , $95 \% \mathrm{Cl}:[0.043,0.304], \mathrm{Z}=2.60, \mathrm{P}<0.05)$ and child patients (MD: $0.192,95 \% \mathrm{Cl}$ : $[0.040,0.343], \mathrm{Z}=2.480, \mathrm{P}<0.05)$ with ASD, but not change in adolescent (MD: $0.208,95 \%$ Cl: $[-0.201,0.618], Z=0.998, P>0.05)$ and adult patients (MD: $0.126,95 \%$ Cl: $[-0.040,0.291], Z=1.488, P>0.05)$.

\subsection{2 subgroup analysis by gender}

To explore the relationship between sex and amygdala volume, a subgroup analysis by gender was conducted. We divided the patients into three groups according to sex: (1) male, (2) female and (3) other unclassified group. Our results show that the volume of the left amygdala not change in male (MD: 0.101, $95 \% \mathrm{Cl}:[-0.031,0.233], Z=1.498, \mathrm{P}>0.05)$ and female (MD: $0.067,95 \% \mathrm{Cl}:[-0.023,0.157], \mathrm{Z}=1.452, \mathrm{P}>0.05)$ group (Fig. $5 \mathrm{~A})$. The volume of the right amygdala significantly increased in female group (MD: $0.099,95 \% \mathrm{Cl}:[0.019,0.178], \mathrm{Z}=2.439, \mathrm{P}<0.05)$, but not change in male group (MD: $0.117,95 \% \mathrm{Cl}$ : $[-0.014,0.248], Z=1.755, P>0.05)$. (Fig. 5B)

\section{Discussion}

The present meta-analysis demonstrates that the volume of the right amygdala significantly increased in patients with ASD. The structural abnormality of the right amygdala may lead to less microstructural integrity in the right amygdala-cortical connections, which are associated with an increased severity of the autism phenotype [44]. The same results were found on the left side, but the results were not robust and greatly affected by heterogeneity.

Further subgroup analysis by age showed that the volume of the left and right amygdala in patients with ASD significantly increased in young child[34], but not change in adolescence and adulthood compare to control. The amygdala in children with autism is initially larger than normal but does not undergo the age-related increase in volume that takes place in typically developing children[48]. The development trajectory of amygdala in ASD drastically deviated from the typical developmental. Anomalous enlargement of the amygdala may be caused by an excessive increase in the number of neurons. Avino et al.'s[15]study found that individuals with autism initially have excess numbers of mature neurons in the amygdala during early childhood, followed by a marked decline beginning in adolescence. Schumann et al.'s [11] research found that the autistic amygdala appears to undergo an abnormal pattern of postnatal development that includes early enlargement and ultimately a reduced number of neurons. Subgroup analysis by gender showed that the volume of the right amygdala was only significantly increased in female of ASD, not in the male. This may be because female with ASD have higher rates of mood disorders [11]and internalizing problems[51] than male counterparts, So that females with ASD may exhibit more severe social[13, 46, 52, 53]. However, due to female individuals being diagnosed less frequently than male individuals at a roughly 1 to 4 rates, female patients are less common, only 3 studies were included in female analysis (14 studies were not reported the volume of amygdala by gender), the results need to be further strengthened by including more literature.

Our results did not entirely consistent with previous analysis[54], which not found significant difference in size of the amygdala between ASD and control. Compared to Stanfield's reports[54], our study had greater statistical power, 28 studies were including in our study and 6 studies in Stanfield et al. Additionally, we performed a sensitivity analysis to ensure the robustness and reliability of the results. However, our research also has some limitations: (1) The number of original studies and the sample size included in the meta-analysis are relatively small, especially in the subgroup analysis. Future studies on gender and age are needed to further corroborate our findings. (2) Heterogeneity, which can greatly affect the conclusions of meta-analysis, was high in our analysis. In addition to age and gender mentioned above, the other influencing factors such as clinical subtypes of ASD, co-occurring other psychopathology disorder and pharmacological interventions are also contributing to the high heterogeneity of ASD [55,56]. First, ASD is a heterogeneous neurodevelopmental disorder, including autism, Asperger's Syndrome (AS), Pervasive Developmental Disorder-Not Otherwise Specified (PDD-NOS), and other subtypes[57]. Both phenotypic heterogeneity and variability in clinical presentation of symptoms of each subtype may contribute to the high heterogeneity[58]. Second, co-occurring other psychopathology disorder is also the source of heterogeneity. Across childhood and adolescence, an estimated $70-80 \%$ of individuals with ASD have at least one co-occurring psychiatric condition, and approximately $40 \%$ have 2 or more[59,60]. Co-occurring other psychopathology disorder such as anxiety and attention-deficit/hyperactivity disorder (ADHD) may affect the patterns of amygdala development [40,61]. Third, pharmacological interventions are also the reasons for the increase in heterogeneity. Despite a lack of pharmacological treatment options for ASD core symptoms, the prevalence of psychopharmacotherapy and polypharmacy in ASD patients is considerable, which is probably due to the treatment of non-core ASD symptoms and psychiatric comorbidities. Prior to pharmacological interventions likely altering neural circuitry and possibly masking the true underlying neural basis of ASD. However, there are simply no enough studies to carry out the corresponding subgroup analysis. (3) Asymmetric funnel plots were found in our analysis. Contour-enhanced funnel plots were used to identify sources of asymmetry. Our study might suggest that the asymmetry of funnel plot was caused by significant heterogeneity which derived from 4 articles[28, 37, 39, 43], rather than publication bias. The heterogeneity of these studies is closely related to the effect size, and these studies have larger effect size $(0.334-1.015)$ than other studies $(-0.17-0.26)$.

\section{Conclusion}

In conclusion, our results show that the volume of the amygdala significantly increased in young children with ASD. These findings suggest that the volume of the amygdala in young children might be a potential early auxiliary imaging diagnosis method of ASD.

\section{Declarations}

\section{Ethics approval and consent to participate}

This article does not contain any studies with human participants or animals performed by any of the authors. No informed consent was required as this article is a review and no individual participants have identifying information. 


\section{Consent for publication}

Not applicable

\section{Competing interests}

The authors declare that there are no competing interests.

\section{Availability of data and materials}

The data that support the findings of this study are available from the first author upon reasonable request.

\section{Conflicts of interest}

The authors declare that they have no conflicts of interest.

\section{Author information}

\section{Affiliations}

Department of Anatomy, School of Basic Medicine, Guizhou Medical University, Guizhou 550025, China

Yunhua Chen

State Key Laboratory of Functions and Applications of Medicinal Plants, Guizhou Medical University, Guizhou 550004, China;

Guizhou Provincial Engineering Technology Research Center for Chemical Drug R\&D

Min Wen and Bo Zhou

\section{Contributions}

Yunhua Chen and Xiaoli Zhen independently searched the literature, extracted the data and analyzed the data. Yunhua Chen completed the first draft of the paper, and Professor Min Wen and Professor Bo Zhou revised and polished the paper.Xue Wang, Ling Yao, Jinggang Peng, Wanxia Liu, Yibu Liu, Xuehui Yan, Yi Zhang assisted.All authors read and approved the final manuscript.

\section{Corresponding author}

Correspondence to Min Wen and Bo Zhou.

\section{Funding}

The authors acknowledge the financial support from PhD research startup foundation of Guizhou medical University (J [2020]008, J [2020]010), Science Technology Foundation Guizhou Provincial Health Commission (gzwkj2021-520), Guizhou Provincial Engineering Laboratory for Chemical Drug R\&D and Guizhou High-School Engineering Research Center for Medicinal Chemistry.

\section{Acknowledgment}

Not applicable

\section{References}

1. Christensen DL, Baio J, Van Naarden BK, Bilder D, Charles J, Constantino JN, et al. Prevalence and Characteristics of Autism Spectrum Disorder Among Children Aged 8 Years-Autism and Developmental Disabilities Monitoring Network, 11 Sites, United States, 2012. MMWR Surveill Summ. 2016;65(3):1-23. http://doi.org/10.15585/mmwr.ss6503a1

2. Shou XJ, Xu XJ, Zeng XZ, Liu Y, Yuan HS, Xing Y, et al. A Volumetric and Functional Connectivity MRI Study of Brain Arginine-Vasopressin Pathways in Autistic Children. Neurosci Bull. 2017;33(2):130-42. http://doi.org/10.1007/s12264-017-0109-2

3. Loomes R, Hull L, Mandy W. What Is the Male-to-Female Ratio in Autism Spectrum Disorder? A Systematic Review and Meta-Analysis. J Am Acad Child Adolesc Psychiatry. 2017;56(6):466-74. http://doi.org/10.1016/j.jaac.2017.03.013 
4. Lord C, Brugha TS, Charman T, Cusack J, Dumas G, Frazier T, et al. Autism spectrum disorder. Nat Rev Dis Primers. 2020;6(1):5. http://doi.org/10.1038/s41572-019-0138-4

5. Moessnang C, Baumeister S, Tillmann J, Goyard D, Charman T, Ambrosino S, et al. Social brain activation during mentalizing in a large autism cohort: the Longitudinal European Autism Project. Mol Autism. 2020;11(1).

6. Kim SY, Choi US, Park SY, Oh SH, Yoon HW, Koh YJ, et al. Abnormal activation of the social brain network in children with autism spectrum disorder: an FMRI study. Psychiatry Investig. 2015;12(1):37-45. http://doi.org/10.4306/pi.2015.12.1.37

7. Sato W, Uono S. The atypical social brain network in autism: advances in structural and functional MRI studies. Curr Opin Neurol. 2019;32(4):617-21. http://doi.org/10.1097/WC0.0000000000000713

8. Baron-Cohen S, Ring HA, Bullmore ET, Wheelwright S, Ashwin C, Williams SC. The amygdala theory of autism. Neurosci Biobehav Rev. 2000;24(3):355-64. http://doi.org/10.1016/s0149-7634(00)00011-7

9. Bickart KC, Dickerson BC, Barrett LF. The amygdala as a hub in brain networks that support social life. Neuropsychologia. 2014;63:235-48. http://doi.org/10.1016/j.neuropsychologia.2014.08.013

10. Sparks BF, Friedman SD, Shaw DW, Aylward EH, Echelard D, Artru AA, et al. Brain structural abnormalities in young children with autism spectrum disorder. Neurology. 2002;59(2):184-92. http://doi.org/10.1212/wnl.59.2.184

11. Schumann CM, Amaral DG. Stereological analysis of amygdala neuron number in autism. J Neurosci. 2006;26(29):7674-9. http://doi.org/10.1523/JNEUROSCI.1285-06.2006

12. Munson J, Dawson G, Abbott R, Faja S, Webb SJ, Friedman SD, et al. Amygdalar volume and behavioral development in autism. Arch Gen Psychiatry. 2006;63(6):686-93. http://doi.org/10.1001/archpsyc.63.6.686

13. Schumann CM, Barnes CC, Lord C, Courchesne E. Amygdala enlargement in toddlers with autism related to severity of social and communication impairments. Biol Psychiatry. 2009;66(10):942-9. http://doi.org/10.1016/j.biopsych.2009.07.007

14. Mosconi MW, Cody-Hazlett H, Poe MD, Gerig G, Gimpel-Smith R, Piven J. Longitudinal study of amygdala volume and joint attention in 2-to 4-year-old children with autism. Arch Gen Psychiatry. 2009;66(5):509-16. http://doi.org/10.1001/archgenpsychiatry.2009.19

15. Avino TA, Barger N, Vargas MV, Carlson EL, Amaral DG, Bauman MD, et al. Neuron numbers increase in the human amygdala from birth to adulthood, but not in autism. P Natl Acad Sci Usa. 2018;115(14).

16. Kemper TL, Bauman M. Neuropathology of infantile autism. J Neuropathol Exp Neurol. 1998;57(7):645-52. http://doi.org/10.1097/00005072-19980700000001

17. Herrero MJ, Velmeshev D, Hernandez-Pineda D, Sethi S, Sorrells S, Banerjee P, et al. Identification of amygdala-expressed genes associated with autism spectrum disorder. Mol Autism. 2020;11(1):39. http://doi.org/10.1186/s13229-020-00346-1

18. Rojas DC, Smith JA, Benkers TL, Camou SL, Reite ML, Rogers SJ. Hippocampus and amygdala volumes in parents of children with autistic disorder. Am J Psychiatry. 2004;161(11):2038-44. http://doi.org/10.1176/appi.ajp.161.11.2038

19. Nacewicz BM, Dalton KM, Johnstone T, Long MT, McAuliff EM, Oakes TR, et al. Amygdala volume and nonverbal social impairment in adolescent and adult males with autism. Arch Gen Psychiatry. 2006;63(12):1417-28. http://doi.org/10.1001/archpsyc.63.12.1417

20. Murphy CM, Deeley Q, Daly EM, Ecker C, O'Brien FM, Hallahan B, et al. Anatomy and aging of the amygdala and hippocampus in autism spectrum disorder: an in vivo magnetic resonance imaging study of Asperger syndrome. Autism Res. 2012;5(1):3-12. http://doi.org/10.1002/aur.227

21. Naama B, Thomas WF, Lucia P, Nancy JM, Matcheri SK, Allan LR, et al. A preliminary longitudinal volumetric MRI study of amygdala and hippocampal volumes in autism. Progress in Neuropsychopharmacology \& Biological Psychiatry. 2014;48.

22. Corbett BA, Carmean V, Ravizza S, Wendelken C, Henry ML, Carter C, et al. A functional and structural study of emotion and face processing in children with autism. Psychiatry Res. 2009;173(3):196-205. http://doi.org/10.1016/j.pscychresns.2008.08.005

23. Moher D, Liberati A, Tetzlaff J, Altman DG. Preferred reporting items for systematic reviews and meta-analyses: the PRISMA statement. Plos Med. 2009;6(7):e1000097. http://doi.org/10.1371/journal.pmed.1000097

24. Peters JL, Sutton AJ, Jones DR, Abrams KR, Rushton L. Contour-enhanced meta-analysis funnel plots help distinguish publication bias from other causes of asymmetry. J Clin Epidemiol. 2008;61(10):991-6. http://doi.org/10.1016/j.jclinepi.2007.11.010

25. Sterne JA, Sutton AJ, loannidis JP, Terrin N, Jones DR, Lau J, et al. Recommendations for examining and interpreting funnel plot asymmetry in metaanalyses of randomised controlled trials. BMJ. 2011;343:d4002. http://doi.org/10.1136/bmj.d4002

26. Chaimani A, Mavridis D, Salanti G. A hands-on practical tutorial on performing meta-analysis with Stata. Evid Based Ment Health. 2014;17(4):111-6. http://doi.org/10.1136/eb-2014-101967

27. Peters JL, Sutton AJ, Jones DR, Abrams KR, Rushton L. Performance of the trim and fill method in the presence of publication bias and between-study heterogeneity. Stat Med. 2007;26(25):4544-62. http://doi.org/10.1002/sim.2889

28. Boucher J, Cowell P, Howard M, Broks P, Farrant A, Roberts N, et al. A combined clinical, neuropsychological, and neuroanatomical study of adults with high functioning autism. Cogn Neuropsychiatry. 2005;10(3):165-213. http://doi.org/10.1080/13546800444000038

29. Pierce K, Muller RA, Ambrose J, Allen G, Courchesne E. Face processing occurs outside the fusiform 'face area' in autism: evidence from functional MRI. Brain. 2001;124(Pt 10):2059-73. http://doi.org/10.1093/brain/124.10.2059

30. Haznedar MM, Buchsbaum MS, Wei TC, Hof PR, Cartwright C, Bienstock CA, et al. Limbic circuitry in patients with autism spectrum disorders studied with positron emission tomography and magnetic resonance imaging. Am J Psychiatry. 2000;157(12):1994-2001.

http://doi.org/10.1176/appi.ajp.157.12.1994

Page 10/16 
31. Palmen SJ, Durston S, Nederveen H, Van Engeland H. No evidence for preferential involvement of medial temporal lobe structures in high-functioning autism. Psychol Med. 2006;36(6):827-34. http://doi.org/10.1017/S0033291706007215

32. Schumann CM, Hamstra J, Goodlin-Jones BL, Lotspeich LJ, Kwon H, Buonocore MH, et al. The amygdala is enlarged in children but not adolescents with autism; the hippocampus is enlarged at all ages. The Journal of neuroscience : the official journal of the Society for Neuroscience. 2004;24(28).

33. Groen W, Teluij M, Buitelaar J, Tendolkar I. Amygdala and hippocampus enlargement during adolescence in autism. J Am Acad Child Adolesc Psychiatry. 2010;49(6):552-60. http://doi.org/10.1016/j.jaac.2009.12.023

34. Kim JE, Lyoo IK, Estes AM, Renshaw PF, Shaw DW, Friedman SD, et al. Laterobasal amygdalar enlargement in 6- to 7-year-old children with autism spectrum disorder. Arch Gen Psychiatry. 2010;67(11):1187-97. http://doi.org/10.1001/archgenpsychiatry.2010.148

35. O'Brien FM, Page L, O'Gorman RL, Bolton P, Sharma A, Baird G, et al. Maturation of limbic regions in Asperger syndrome: a preliminary study using proton magnetic resonance spectroscopy and structural magnetic resonance imaging. Psychiatry Res. 2010;184(2):77-85. http://doi.org/10.1016/j.pscychresns.2010.08.007

36. Cheung C, McAlonan GM, Fung YY, Fung G, Yu KK, Tai KS, et al. MRI study of minor physical anomaly in childhood autism implicates aberrant neurodevelopment in infancy. Plos One. 2011;6(6):e20246. http://doi.org/10.1371/journal.pone.0020246

37. Hazlett HC, Poe MD, Lightbody AA, Gerig G, Macfall JR, Ross AK, et al. Teasing apart the heterogeneity of autism: Same behavior, different brains in toddlers with fragile X syndrome and autism. J Neurodev Disord. 2009;1(1):81-90. http://doi.org/10.1007/s11689-009-9009-8

38. Dziobek I, Bahnemann M, Convit A, Heekeren HR. The role of the fusiform-amygdala system in the pathophysiology of autism. Arch Gen Psychiatry. 2010;67(4):397-405. http://doi.org/10.1001/archgenpsychiatry.2010.31

39. Zhu Z, Fang X, Chen H, Zhu X, Zhang L, Zhai X, et al. Alterations in volumes and MRI features of amygdala in Chinese autistic preschoolers associated with social and behavioral deficits. Brain Imaging Behav. 2018;12(6):1814-21. http://doi.org/10.1007/s11682-018-9853-9

40. Herrington JD, Maddox BB, Kerns CM, Rump K, Worley JA, Bush JC, et al. Amygdala Volume Differences in Autism Spectrum Disorder Are Related to Anxiety. J Autism Dev Disord. 2017;47(12):3682-91. http://doi.org/10.1007/s10803-017-3206-1

41. Lucibello S, Verdolotti T, Giordano FM, Lapenta L, Infante A, Piludu F, et al. Brain morphometry of preschool age children affected by autism spectrum disorder: Correlation with clinical findings. Clin Anat. 2019;32(1):143-50. http://doi.org/10.1002/ca.23252

42. Maier S, Tebartz VEL, Beier D, Ebert D, Fangmeier T, Radtke M, et al. Increased hippocampal volumes in adults with high functioning autism spectrum disorder and an IQ>100: A manual morphometric study. Psychiat Res. 2015;234(1).

43. Margaret S, Jodene GF, Jesse B, David CZ. Magnetic resonance imaging volumetric findings in children with Asperger syndrome, nonverbal learning disability, or healthy controls. J Clin Exp Neuropsyc. 2013;35(5).

44. Gibbard CR, Ren J, Skuse DH, Clayden JD, Clark CA. Structural connectivity of the amygdala in young adults with autism spectrum disorder. Hum Brain Mapp. 2018;39(3):1270-82. http://doi.org/10.1002/hbm.23915

45. J, C., LI, X., CX, W., JG, L., \& B, Z. (2016). Analysis of cerebral gray matter structural changes in autistic children by IBASPM. . J Chin Clin Med Imaging, 27(5), 309-313.

46. Nordahl CW, losif AM, Young GS, Hechtman A, Heath B, Lee JK, et al. High Psychopathology Subgroup in Young Children With Autism: Associations With Biological Sex and Amygdala Volume. J Am Acad Child Adolesc Psychiatry. 2020;59(12):1353-63. http://doi.org/10.1016/j.jaac.2019.11.022

47. Trontel HG, Duffield TC, Bigler ED, Abildskov TJ, Froehlich A, Prigge MB, et al. Mesial temporal lobe and memory function in autism spectrum disorder: an exploration of volumetric findings. J Clin Exp Neuropsychol. 2015;37(2):178-92. http://doi.org/10.1080/13803395.2014.997677

48. Xu Q, Zuo C, Liao S, Long Y, Wang Y. Abnormal development pattern of the amygdala and hippocampus from childhood to adulthood with autism. J Clin Neurosci. 2020;78:327-32. http://doi.org/10.1016/j.jocn.2020.03.049

49. Rynkiewicz A, Lucka I. Autism spectrum disorder (ASD) in girls. Co-occurring psychopathology. Sex differences in clinical manifestation. Psychiatr Pol. 2018;52(4):629-39. http://doi.org/10.12740/PP/OnlineFirst/58837

50. Kreiser NL, White SW. ASD Traits and Co-occurring Psychopathology: The Moderating Role of Gender. J Autism Dev Disord. 2015;45(12):3932-8. http://doi.org/10.1007/s10803-015-2580-9

51. Solomon M, Miller M, Taylor SL, Hinshaw SP, Carter CS. Autism symptoms and internalizing psychopathology in girls and boys with autism spectrum disorders. J Autism Dev Disord. 2012;42(1):48-59. http://doi.org/10.1007/s10803-011-1215-z

52. Evans SC, Boan AD, Bradley C, Carpenter LA. Sex/Gender Differences in Screening for Autism Spectrum Disorder: Implications for Evidence-Based Assessment. J Clin Child Adolesc Psychol. 2019;48(6):840-54. http://doi.org/10.1080/15374416.2018.1437734

53. Wang S, Deng H, You C, Chen K, Li J, Tang C, et al. Sex Differences in Diagnosis and Clinical Phenotypes of Chinese Children with Autism Spectrum Disorder. Neurosci Bull. 2017;33(2):153-60. http://doi.org/10.1007/s12264-017-0102-9

54. Stanfield AC, McIntosh AM, Spencer MD, Philip R, Gaur S, Lawrie SM. Towards a neuroanatomy of autism: a systematic review and meta-analysis of structural magnetic resonance imaging studies. Eur Psychiatry. 2008;23(4):289-99. http://doi.org/10.1016/j.eurpsy.2007.05.006

55. Lenroot RK, Yeung PK. Heterogeneity within Autism Spectrum Disorders: What have We Learned from Neuroimaging Studies? Front Hum Neurosci. 2013;7:733. http://doi.org/10.3389/fnhum.2013.00733

56. Masi A, DeMayo MM, Glozier N, Guastella AJ. An Overview of Autism Spectrum Disorder, Heterogeneity and Treatment Options. Neurosci Bull. 2017;33(2):183-93. http://doi.org/10.1007/s12264-017-0100-y

57. Lord C, Bishop SL. Recent advances in autism research as reflected in DSM-5 criteria for autism spectrum disorder. Annu Rev Clin Psychol. 2015;11:53-70. http://doi.org/10.1146/annurev-clinpsy-032814-112745

Page $11 / 16$ 
58. Steiner AM, Goldsmith TR, Snow AV, Chawarska K. Practitioner's guide to assessment of autism spectrum disorders in infants and toddlers. J Autism Dev Disord. 2012;42(6):1183-96. http://doi.org/10.1007/s10803-011-1376-9

59. Houghton R, Ong RC, Bolognani F. Psychiatric comorbidities and use of psychotropic medications in people with autism spectrum disorder in the United States. Autism Res. 2017;10(12):2037-47. http://doi.org/10.1002/aur.1848

60. Rosen TE, Mazefsky CA, Vasa RA, Lerner MD. Co-occurring psychiatric conditions in autism spectrum disorder. Int Rev Psychiatry. 2018;30(1):40-61. http://doi.org/10.1080/09540261.2018.1450229

61. Tajima-Pozo K, Yus M, Ruiz-Manrique G, Lewczuk A, Arrazola J, Montanes-Rada F. Amygdala Abnormalities in Adults With ADHD. J Atten Disord. 2018;22(7):671-8. http://doi.org/10.1177/1087054716629213

\section{Figures}

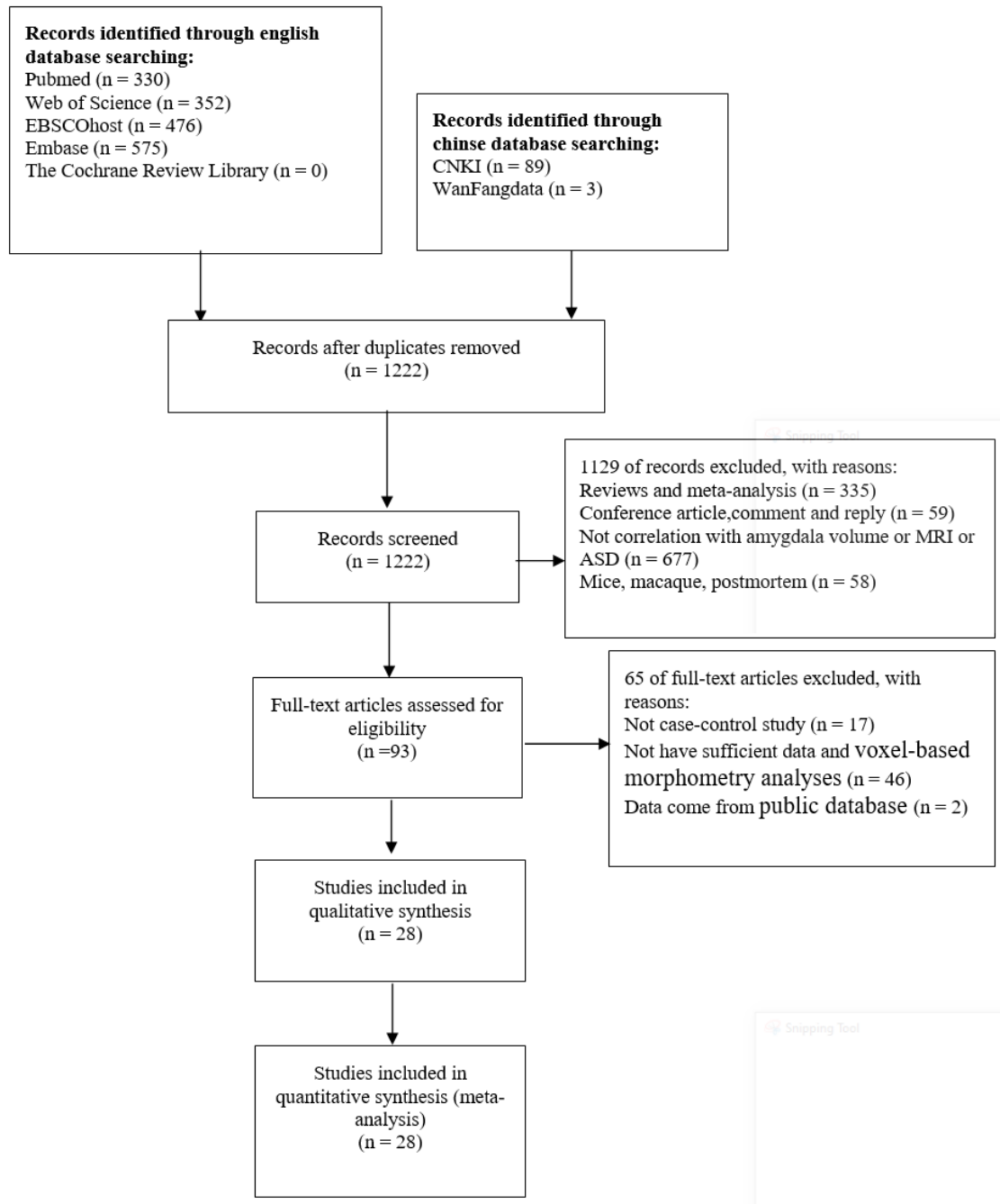

Figure 1

PRISMA 2009 Flow Diagram 

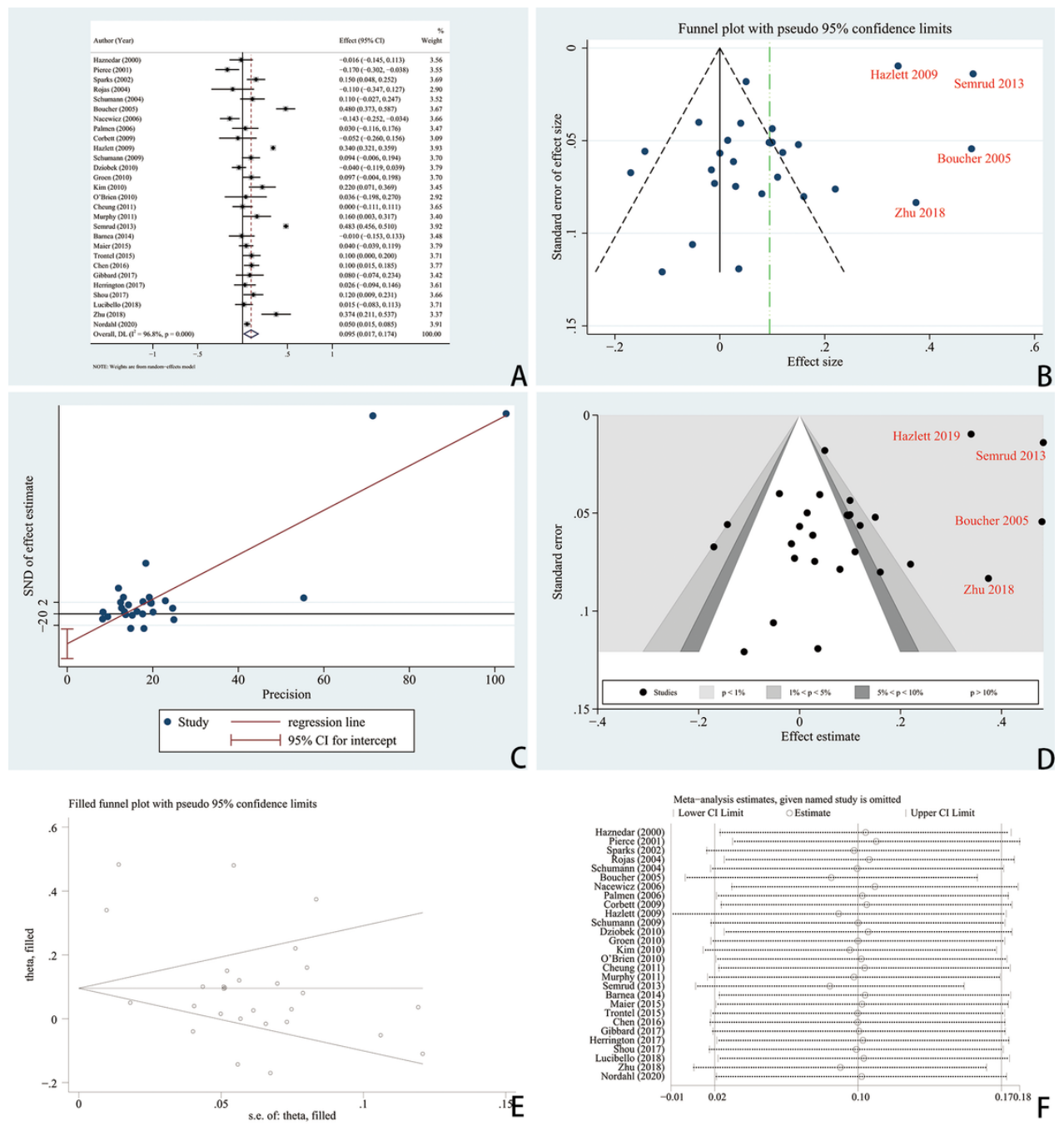

\section{Figure 2}

28 studies (included 1011 ASD and 823 controls) were used in this analysis. These studies had significant heterogeneity (I2 = 96.8\%, 95\% Cl: [82.3\%, 98.7\%]; $\mathrm{H}$ $\left.=5.582,95 \% \mathrm{Cl}:[2.374,8.836], \mathrm{tau}^{2}=0.0406\right)$, so a random-effect model was used to calculate pooled WMD. Forest plot (Fig $\left.2 \mathrm{~A}\right)$ showed that the diamond was on the right side of the vertical line and did not intersect with the line, which demonstrates a significantly increase the volume of the left amygdala in patients with ASD compared to control (WMD: $0.095,95 \% \mathrm{Cl}$ : [0.017, 0.174], Z = 2.379, P< 0.05). The funnel plot (fig 2B) was asymmetrical, as assessed by visual inspection and Egger's meta-regression analysis ( $\mathrm{p}<0.001,95 \% \mathrm{Cl}$ : $[-7.79,-2.63]$, fig $2 \mathrm{C}$ ). To differentiating asymmetry due to publication bias from that due to other factors, the contour-enhanced funnel plot (fig 2D) was used. 

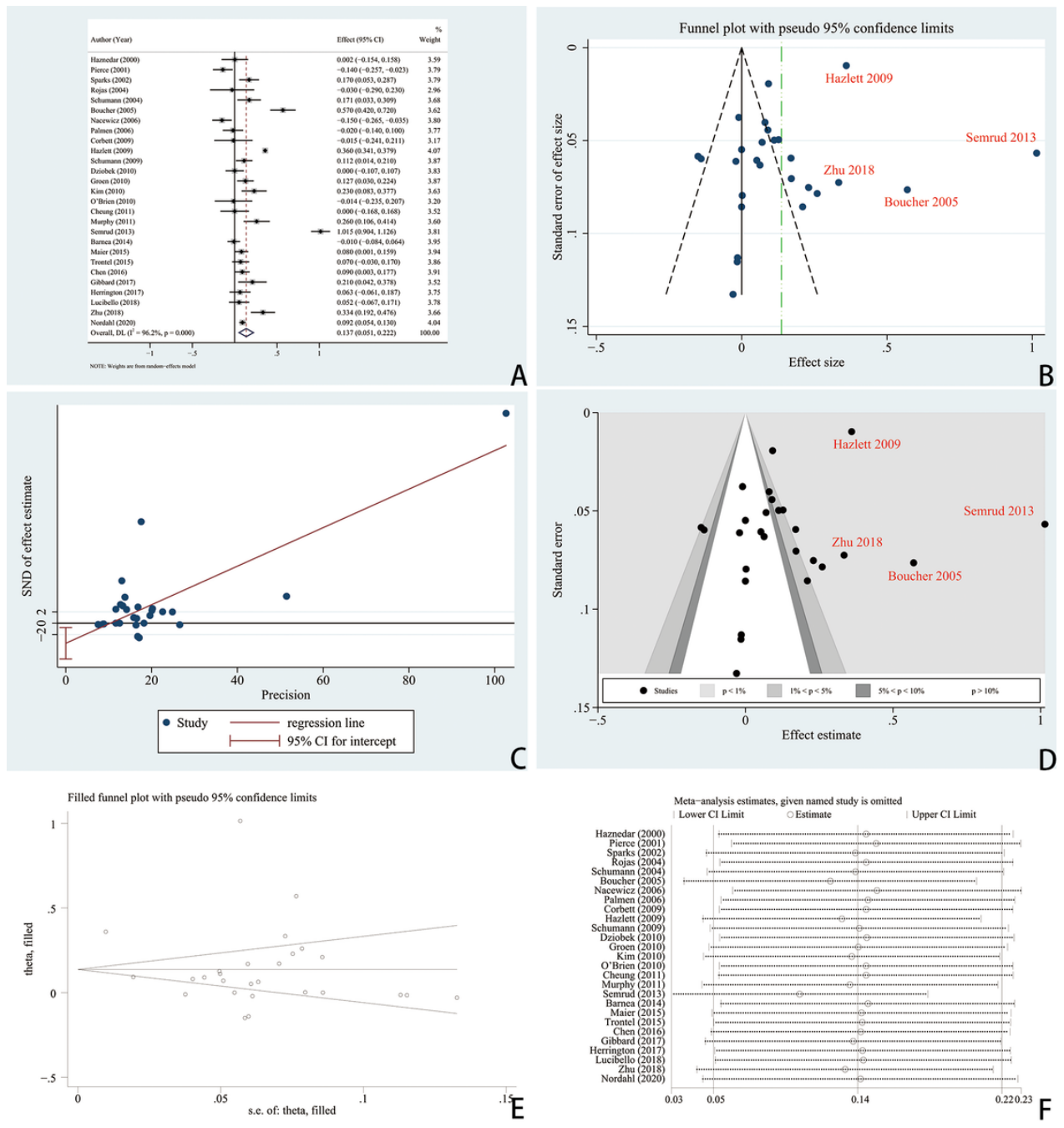

\section{Figure 3}

27 studies (included 996 ASD and 809 controls) were used in this analysis. These studies had significant heterogeneity ( $12=96.2 \%, 95 \% \mathrm{Cl}:[79.7 \%, 98.5 \%] ; \mathrm{H}=$ $5.127,95 \% \mathrm{Cl}:[2.218,8.075]$, tau $\left.{ }^{2}=0.0469\right)$, so a random-effect model was used to calculate pooled WMD. Forest plot (Fig $\left.3 \mathrm{~A}\right)$ showed that the diamond was on the right side of the vertical line and did not intersect with the line, which demonstrates a significantly increase the volume of the right amygdala in patients with ASD compared to control (WMD: $0.137,95 \% \mathrm{Cl}$ : [0.051, 0.222], Z = 3.132, P< 0.001). The funnel plot (fig 3B) was asymmetrical, as assessed by visual inspection of the plot and by Egger's meta-regression analysis ( $p=0.016<0.05,95 \% \mathrm{Cl}$ : $[-6.30,-0.75]$, fig $3 \mathrm{C})$. To differentiating asymmetry due to publication bias from that due to other factors, the contour-enhanced funnel plot (fig 3D) was used. 


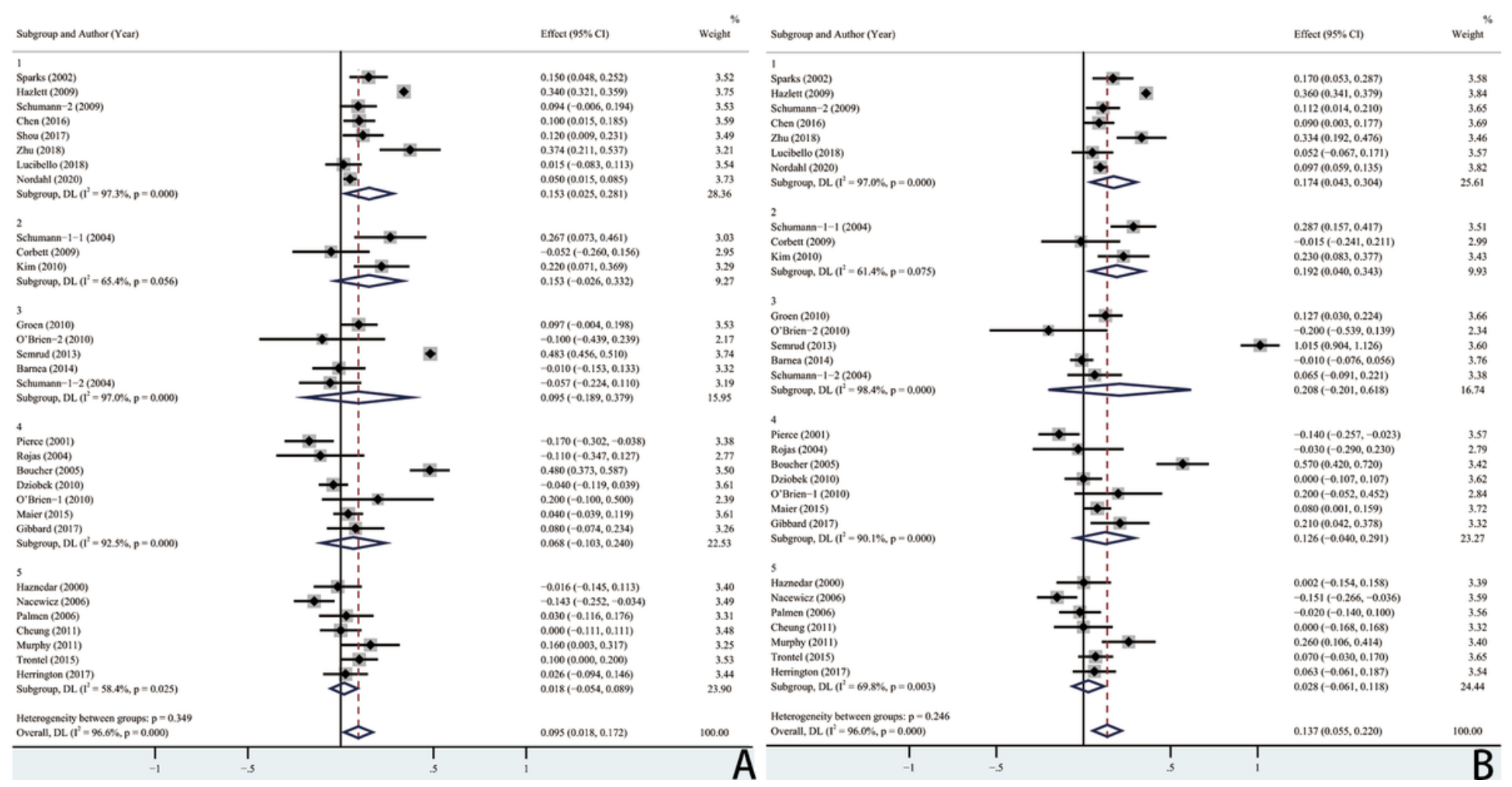

\section{Figure 4}

To explore the relationship between age and amygdala volume, a subgroup analysis by age was conducted. We divided all studies into five groups according to age: (1) young children (0-6 years), (2) children (6-12 years), (3) adolescent (12-18 years), (4) adult (>18 years) and (5) other group. Our results show that the volume of the left amygdala (fig 4A) significantly increase in young child patients with ASD (MD: $0.153,95 \% \mathrm{Cl}:[0.025,0.281], \mathrm{Z}=2.341, \mathrm{P}<0.05$ ), but not change in child (MD: $0.153,95 \% \mathrm{Cl}:[-0.026,0.332], \mathrm{Z}=1.672, \mathrm{P}>0.05)$, adolescent (MD: 0.095, 95\% Cl: $[-0.189,0.379], Z=0.654, \mathrm{P}>0.05)$ and adult (MD: $0.068,95 \% \mathrm{Cl}:[-0.103,0.240], \mathrm{Z}=0.781, \mathrm{P}>0.05)$ ASD patient. The volume of the right amygdala (fig 4B) significantly increased in young child (MD: 0.174 , 95\% Cl: $[0.043,0.304], Z=2.60, P<0.05)$ and child patients (MD: 0.192, 95\% Cl: [0.040, 0.343], Z = 2.480, $\mathrm{P}<0.05$ ) with ASD, but not change in adolescent (MD: $0.208,95 \% \mathrm{Cl}:[-0.201,0.618], Z=0.998, P>0.05)$ and adult patients (MD: $0.126,95 \%$ Cl: $[-0.040,0.291], Z=1.488, P>0.05)$.

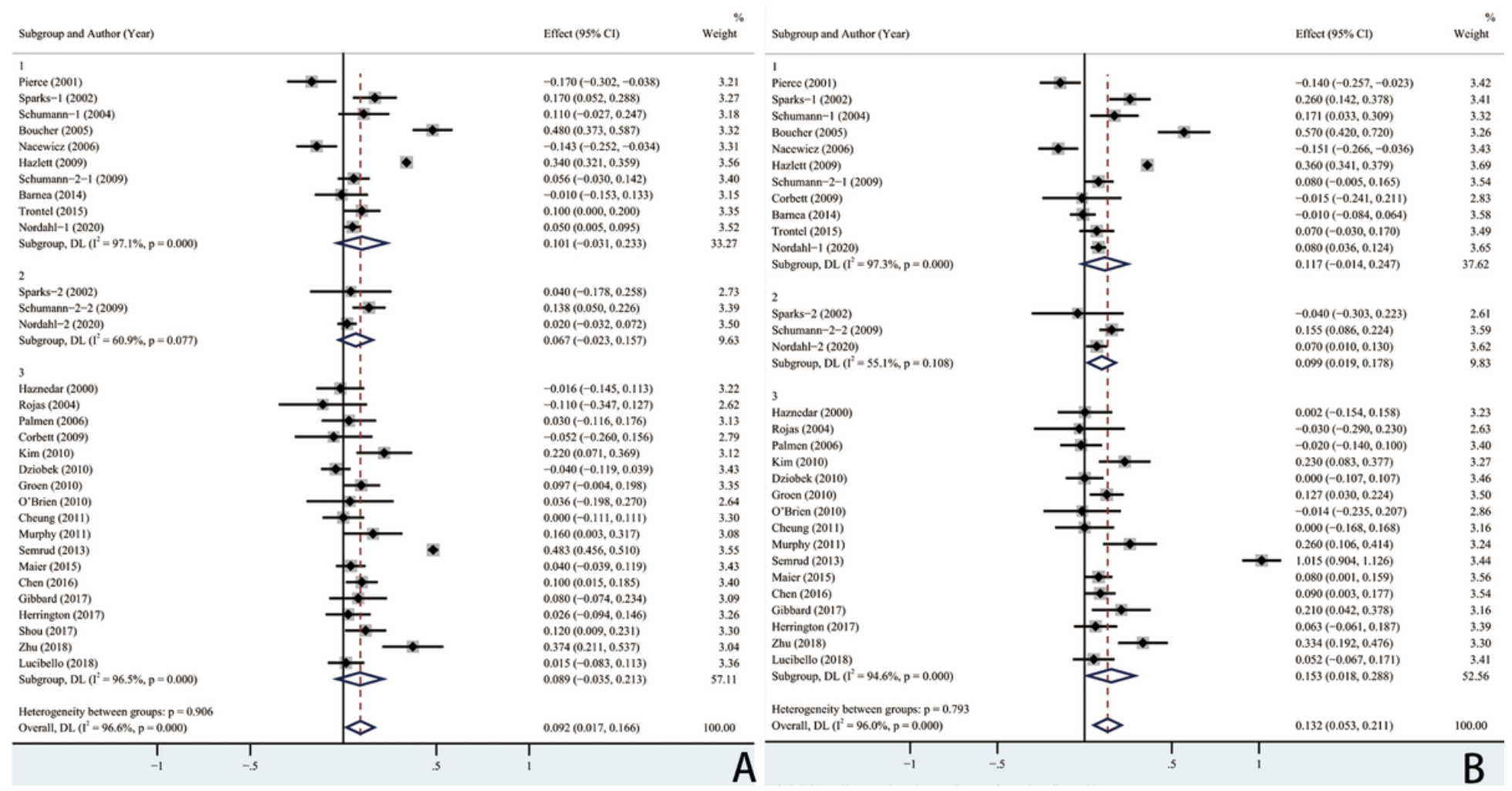

Figure 5 
To explore the relationship between sex and amygdala volume, a subgroup analysis by gender was conducted. We divided the patients into three groups according to sex: (1) male, (2) female and (3) other unclassified group. Our results show that the volume of the left amygdala not change in male (MD: 0.101, $95 \% \mathrm{Cl}:[-0.031,0.233], \mathrm{Z}=1.498, \mathrm{P}>0.05)$ and female (MD: $0.067,95 \% \mathrm{Cl}:[-0.023,0.157], \mathrm{Z}=1.452, \mathrm{P}>0.05)$ group (fig $5 \mathrm{~A})$. The volume of the right amygdala significantly increased in female group (MD: 0.099, 95\% Cl: [0.019, 0.178], Z $=2.439, \mathrm{P}<0.05)$, but not change in male group (MD: $0.117,95 \% \mathrm{Cl}$ : $[-0.014,0.248], Z=1.755, P>0.05)$. (fig 5B) 